\title{
Emergency Thoracotomy Saves Lives in a Scandinavian Hospital Setting
}

\author{
Andreas Saxlund Pahle, Bastian Løe Pedersen, Nils Oddvar Skaga, MD, PhD, and Johan Pillgram-Larsen, MD
}

\begin{abstract}
Background: Emergency thoracotomy (ET) is a life-saving procedure used to control hemorrhage and relieve cardiac tamponade. It has been in routine use at Ulleval University Hospital since 1987. Our objective was to see the outcome of patients subjected to ET in recent times.

Methods: One hundred and nine consecutive ET performed in our emergency department during a 6-year period were analyzed. Data were drawn from the hospital's trauma registry. Demographics, mechanism of injury, anatomic injuries, physiologic status, interventions, time lapse, and outcome 30 days after injury were registered prospectively.

Results: Ten of 27 patients with penetrating (37\%) and 10 of 82 patients with blunt injuries $(12 \%)$ survived, giving a total survival of $18 \%$. Median (quartiles) for the following parameters were Injury Severity Score 38 (26-50), Revised Trauma Score $1.3(0-3.9)$, Glasgow Coma Scale score 3 $(3-6)$, and probability of survival $0.06(0.001-0.22)$. Survivors from penetrating injuries had significantly lower Injury Severity Score ( 25 vs. 34, $p=$ 0.003 ), higher Revised Trauma Score (3.92 vs. 0.00, $p<0.001$ ), higher Glasgow Coma Scale score ( 8 vs. $3, p<0.001)$, and higher probability of survival $(0.74$ vs. $0.01, p<0.001)$ than nonsurvivors. Conversely, no such differences were found for patients with blunt injury. Multiple logistic regression analysis failed to reveal any predictors of survival.

Conclusion: An overall survival of $18 \%$ suggests that ET is a life saving procedure. It is difficult to find good predictors of survival from logistic regression analysis. It should, for a trained trauma team, be a liberal attitude toward performing the procedure on the agonal patient.
\end{abstract}

Key Words: Trauma, Resuscitation, Emergency thoracotomy.

(J Trauma. 2009;XX: 000-000)

$\mathrm{E}_{\mathrm{f}}^{\mathrm{n}}$ mergency thoracotomy (ET) is a surgical procedure performed in the emergency room for the critically injured patient presenting with hemorrhage or cardiac tamponade. Since its reintroduction as a salvaging tool for patients with chest wounds in the late $1960 \mathrm{~s},{ }^{1}$ indications for ET now include aortic cross clamping for exsanguinated patients after abdominal injury; pericardial decompression; intrathoracic hemorrhage control; open cardiac massage; and correction of bronchovenous air embolism. ${ }^{1-3}$ Over the last decades, the

Submitted for publication September 30, 2008.

Accepted for publication February 6, 2009.

Copyright (C) 2009 by Lippincott Williams \& Wilkins

From the Department of Cardiothoracic Surgery (A.S.P., B.L.P.), Ulleval University Hospital, Oslo, Norway; Departments of Anesthesia (N.O.S.) and Cardiothoracic Surgery (J.P.-L.), Ulleval University Hospital, Oslo, Norway.

The first two authors contributed equally to this work.

Address for reprints: Johan Pillgram-Larsen, MD, Department of Cardiothoracic Surgery, Ulleval University Hospital, 0407 Oslo, Norway; email: pillgram@ hotmail.com.

DOI: 10.1097/TA.0b013e3181a5ec54 outcome of the method has been critically analyzed.1,2,4-11 The current notion supports a rather selective approach to the procedure. ${ }^{1,4,9,11}$ In accordance with this, the recently published 8th edition of the Advanced Trauma Life Support (ATLS) course concluded that ET as a resuscitative intervention for patients with blunt injuries and cardiac arrest is rarely effective. ${ }^{11}$

There are few reports on ET outcome from Europe in general, and from Scandinavia, in particular. This paucity requires US guidelines to set precedence for many trauma protocols in Norway, including ET. In a recent study from a relatively busy Norwegian trauma hospital, Stavanger University Hospital, it was stated that outcome of ET was dismal. ${ }^{9}$ They reported zero survivors of 10 patients subjected to ET during a 5-year period. ${ }^{9}$ Reevaluation of the decisionmaking process for the procedure was proposed.

Ulleval University Hospital (UUH) is Norway's largest hospital. The aim of this study was to examine the outcome of 109 patients subjected to ET in the emergency department (ED) at UUH. We hypothesized that overall survival after the procedure in the largest trauma center in Norway justifies the use of this surgical intervention.

\section{MATERIALS AND METHODS}

\section{Population and Study Database}

UUH is the major trauma hospital for 540,000 citizens and the trauma referral center for 2.5 million people. Annually, roughly 1,200 patients are entered in the hospital-based trauma registry. Of these, $40 \%$ are seriously injured, i.e., ISS $>15$. Ninety percent are exposed to blunt and $10 \%$ to penetrating injuries.

The inclusion period was from January 2001 to September 2007. All data have been prospectively collected during the hospital stay. Inclusion criteria were all patients who underwent ET in the ED in the study period. Urgent thoracotomies in the operating room are not included. Information extracted from the trauma registry has been supplemented with data from the patient administrative system. The representative for The Norwegian Data Inspectorate at UUH has approved the study.

\section{The Trauma Team}

UUH has a formalized trauma admitting system with $\sim 1,000$ trauma team activations a year. The trauma team consists of the most senior resident in general surgery (team leader), the resident in cardiothoracic surgery, a consultant anesthesiologist, nurse anesthetist, operation room nurse, ra- 
diographer, laboratory technician, and a nurse coordinator. Other resources are requested when needed. Trauma team activation criteria are obvious severe injury, circulatory or respiratory instability, reduced level of consciousness, highenergy trauma, or other situations with a high index of concern. The trauma team leader has passed ATLS examination, has attended the practical war surgery and traumatology course, and has been supervised in ET in the pathology lab.

\section{ET at UUH}

Emergency thoracatomies have been performed since 1987 at UUH. ${ }^{12}$ Indications for the procedure are listed in the hospitals trauma manual ${ }^{3}$ : (1) unresponsive patient with penetrating injury who has shown signs of life (SOL) during transport or at the scene of crash; (2) exsanguinated patients without immediate response to fluid resuscitation; (3) obviously large abdominal bleeding and decreasing blood pressure with no response to fluid resuscitation before laparotomy. Contraindications are (1) patients subjected to blunt trauma without SOL at admission and (2) patients above 60 years without SOL at admission.

Our ET technique includes median sternotomy or anterolateral thoracotomy depending on the suspected location of the injury. Sternotomy is the preferred technique for relieving cardiac tamponade, penetrating injuries to the mediastinal compartment, and penetrating injuries to the supraclavicular area. Anterolateral thoracotomy is preferred for cross clamping of the thoracic aorta, open cardiac massage, and penetrating injuries to either of the two pulmonal compartments. Patients in which both techniques are used are pooled under the label "combined" in the subsequent analysis. The trauma team leader makes the decision for any procedure during a trauma call. At UUH, all team leaders must be able to perform an ET. The cardiothoracic resident is part of the team and is often the person performing the procedure.

\section{Injury Coding}

The ISS ${ }^{13-15}$ was based on the Abbreviated Injury Scale 1990 Update 98 (AIS 98). ${ }^{16}$ The coding included all accessible information until discharge. The trauma registrar is a registered nurse anesthetist, formally educated in injury coding (AIS 98) through a standard course of the Association for the Advancement of Automotive Medicine in the United States. Physiologic derangement was coded according to Revised Trauma Score (RTS). ${ }^{17}$ In patients who were in general anesthesia or intubated on admission, Glasgow Coma Scale (GCS) score and respiratory rates were scored based on values documented immediately before intubation. ${ }^{18}$ For calculation of probability of survival (PS), we used Trauma and Injury Severity Score methodology with updated coefficients for AIS $90 .{ }^{16,17,19} \mathrm{SOL}$ in the field are defined as having one or more of the following: spontaneous movements, pupillary response, eye movement, spontaneous respirations, or cardiac activity.

\section{Outcome Definition}

Survival status 30 days after injury (alive or dead), whether the patient was discharged from acute care or not, was obtained from patient records and the Norwegian Population Registry. ${ }^{20}$ In Norway, all the residents can be traced by their social security number.

\section{Statistics}

Continuous data are presented as median (quartiles) values. Categorical data are presented as percentages. Between-group tests were made by Mann-Whitney $U$ test and $\chi^{2}$-test for continuous and categorical data, respectively. After univariate analysis, a forward stepwise multiple logistic regression model was conducted to validate outcome (survivor/nonsurvivor). Predictors were stratified according to clinical variables available to the trauma team leader at admission and included GCS score $>3$, SOL at site, ET before/after 30 minutes, dominant mechanism of injury, gender, age, and time of arrival after injury. Interaction terms were generated and included in the model. For all statistical analysis, a two-sided $p$ value of 0.05 was considered significant. All data analyses were undertaken using Statistical Package for the Social Sciences; release 15.0 for Windows (SPSS, Inc., Chicago, IL).

\section{RESULTS}

Of the total 109 patients subjected to ET, 20 (18\%) survived. Baseline characteristics are depicted in Table 1. Anterolateral ET was performed in 74 patients (68\%), median sternotomy in 10 patients $(9 \%)$, and 25 patients $(23 \%)$ underwent both. The injured were predominantly young (median age, 30 years) males (69\%) exposed to blunt injuries (75\%). Median ISS was 38 (quartiles, 26-50), RTS 1.3 (quartiles, 0-3.92), GCS score 3 (quartiles, 3-6), and PS 0.06 (quartiles, 0.001-0.22). Further, the patients were hypotensive (blood pressure, $40 \mathrm{~mm} \mathrm{Hg}$ ) and bradycardic (65 beats per minute). Seventy-nine percent $(n=86)$ showed SOL at

TABLE 1. Baseline Characteristics on All Patients Treated With Emergency Thoracotomy

\begin{tabular}{|c|c|c|}
\hline Variable & $\mathrm{N}=109(\%)$ & Missing \\
\hline Survivors & $20(18 \%)$ & \\
\hline Anterolateral ET & $74(68 \%)$ & \\
\hline Median sternotomy & $10(9 \%)$ & \\
\hline Combined ${ }^{*}$ & $25(23 \%)$ & \\
\hline Age (yr) & $30(24-47)$ & 1 \\
\hline Sex, males & $75(69 \%)$ & \\
\hline Penetrating & $27(25 \%)$ & \\
\hline Blunt & $82(75 \%)$ & \\
\hline ISS & $38(26-50)$ & \\
\hline GCS score & $3(3-6)$ & \\
\hline PS & $0.06(0.001-0.22)$ & \\
\hline RTS & $1.31(0-3.92)$ & \\
\hline Blood pressure $(\mathrm{mm} \mathrm{Hg})$ & $40(0-85)$ & 18 \\
\hline Pulse & $65(0-120)$ & 12 \\
\hline SOL at injury site & $86(79 \%)$ & 2 \\
\hline $\mathrm{ET}<30 \mathrm{~min}$ after injury & $26(24 \%)$ & 26 \\
\hline Arrival ED after injury (min) & $40.5(18-84)$ & 7 \\
\hline ET after initial presentation & $3(1-13)$ & 30 \\
\hline
\end{tabular}


TABLE 2. Patient Characteristics Grouped by Dominating Mechanism of Injury

\begin{tabular}{|c|c|c|c|c|}
\hline Variable & $\begin{array}{l}\text { Penetrating } \\
(\mathrm{n}=27)\end{array}$ & $\begin{array}{c}\text { Blunt } \\
(\mathrm{n}=82)\end{array}$ & $\begin{array}{c}p \\
\text { Value }\end{array}$ & Missing \\
\hline Anterolateral ET & $8(30 \%)$ & $66(80 \%)$ & $<0.001$ & \\
\hline Median sternotomy & $7(26 \%)$ & $3(4 \%)$ & $<0.001$ & \\
\hline Combined* & $12(44 \%)$ & $13(16 \%)$ & $<0.001$ & \\
\hline Age (yr) & $28(24-37)$ & $35(24-48)$ & 0.128 & 1 \\
\hline Sex, males & $23(85 \%)$ & $52(63 \%)$ & 0.034 & \\
\hline Survivor & $10(37 \%)$ & $10(12 \%)$ & 0.004 & \\
\hline ISS & $27(25-38)$ & $43(34-50)$ & 0.005 & \\
\hline PS & $0.03(0.01-0.42)$ & $0.01(0.02-0.21)$ & 0.305 & \\
\hline RTS & $0.29(0.00-3.57)$ & $1.47(0.00-3.92)$ & 0.190 & \\
\hline GCS score & $3(3-7)$ & $3(3-6)$ & 0.918 & \\
\hline $\begin{array}{l}\text { SOL at scene of } \\
\text { accident }\end{array}$ & $17(63 \%)$ & $69(84 \%)$ & 0.042 & 2 \\
\hline $\begin{array}{l}\text { Arrival ED after } \\
\text { injury (min) }\end{array}$ & $25(15-35)$ & $55(20-94)$ & 0.020 & 7 \\
\hline $\begin{array}{l}\text { ET }<30 \text { min after } \\
\text { injury }\end{array}$ & 12 & 14 & 0.003 & $26^{\dagger}$ \\
\hline $\begin{array}{l}\mathrm{ET}>30 \text { min after } \\
\text { injury }\end{array}$ & 9 & 48 & & \\
\hline
\end{tabular}

All values are given as median (quartiles).

* Anterolateral and median sternotomy.

${ }^{\dagger}$ Missing 6 in penetrating group and 20 in blunt group

the scene of accident. Median time span between the patient arriving at the ED and initiating ET was 3 minutes (quartiles, $1-13$ minutes).

\section{Causes of Injury}

The patients were classified according to dominant mechanism of injury: blunt or penetrating (Table 2). Most common causes of injury were motor vehicle collisions $(\mathrm{n}=$ 54) and fall accidents $(\mathrm{n}=22)$ for blunt and stab wounds $(\mathrm{n}=19)$ for penetrating. Those suffering from penetrating injuries were more likely to have a lower ISS $(p=0.005)$ and were less likely to have SOL at the scene of crash $(p=0.042)$ compared with the blunt injury group. Furthermore, victims of penetrating injury arrived earlier to the $\operatorname{ED}(p=0.02)$ and were subjected to ET faster $(p=0.003)$ than the blunt injured group. Patients with penetrating injuries were treated most often with combined ET (median sternotomy and anterolateral) $(44 \%)$, whereas those with blunt injuries were treated most often with anterolateral ET $(80 \%)$.

\section{Outcome}

In Table 3, patients are categorized according to final outcome (survivor/nonsurvivors). Survivors were less severely injured (ISS 26 vs. $42 ; p<0.001$ ) and presented with higher GCS score (6.5 vs. 3.0; $p<0.001)$ than nonsurvivors. No statistical difference was found between the two groups when correlating outcome against technique (anterolateral vs. median sternotomy vs. combined) for ET ( $p=0.954)$, age $(p=0.672)$, gender $(p=0.601)$, observed SOL at site of injury $(p=0.307)$, ET performed within 30 minutes after injury $(p=0.994)$, or arrival time at the emergency room after injury (45 minutes vs. 41 minutes, $p=0.477$ ).
TABLE 3. Patients Treated With Emergency Thoracotomy, Grouped by Outcome

\begin{tabular}{|c|c|c|c|c|}
\hline Variable & $\begin{array}{c}\text { Nonsurvivors } \\
(\mathbf{n}=\mathbf{8 9})\end{array}$ & $\begin{array}{l}\text { Survivors } \\
(\mathrm{n}=\mathbf{2 0})\end{array}$ & $\begin{array}{c}p \\
\text { Value }\end{array}$ & Missing \\
\hline Anterolateral ET & $61(69 \%)$ & $13(65 \%)$ & 0.954 & \\
\hline Median sternotomy & $8(9 \%)$ & $2(10 \%)$ & 0.954 & \\
\hline Combined* & $20(22 \%)$ & $5(25 \%)$ & 0.954 & \\
\hline Age (yr) & $29(24-46)$ & $34(24-48)$ & 0.672 & 1 \\
\hline Sex, males & $60(67 \%)$ & $15(75 \%)$ & 0.601 & \\
\hline Penetrating injury & $17(63 \%)$ & $10(37 \%)$ & 0.004 & \\
\hline Blunt injury & $72(88 \%)$ & $10(12 \%)$ & & \\
\hline RTS score & $0.94(0-3.57)$ & $3.92(2.63-6.03)$ & 0.001 & \\
\hline ISS & $42(29-51)$ & $26(16-38)$ & 0.001 & \\
\hline PS & $0.06(0.01-0.14)$ & $0.54(0.06-0.93)$ & 0.001 & \\
\hline GCS score & $3(3-5)$ & $7(3-12)$ & 0.001 & \\
\hline $\begin{array}{l}\text { SOL at scene of } \\
\text { accident }\end{array}$ & $67(75 \%)$ & $19(95 \%)$ & 0.307 & 2 \\
\hline $\begin{array}{l}\text { Arrival ED after } \\
\text { injury (min) }\end{array}$ & $40(18-84)$ & $45(25-95)$ & 0.477 & 7 \\
\hline $\begin{array}{l}\text { ET }<30 \text { min after } \\
\text { injury }\end{array}$ & 21 & 5 & 0.994 & $26^{\dagger}$ \\
\hline $\begin{array}{l}\text { ET }>30 \text { min after } \\
\text { injury }\end{array}$ & 46 & 11 & & \\
\hline
\end{tabular}

TABLE 4. Patients With Penetrating Injuries Treated With Emergency Thoracotomy, Grouped by Outcome

\begin{tabular}{|c|c|c|c|c|}
\hline Variable & $\begin{array}{l}\text { Nonsurvivors } \\
\qquad(\mathrm{n}=17)\end{array}$ & $\begin{array}{l}\text { Survivors } \\
(n=10)\end{array}$ & $\underset{\text { Value }}{p}$ & Missing \\
\hline Anterolateral ET & $3(18 \%)$ & $5(50 \%)$ & 0.203 & \\
\hline Median sternotomy & $5(29 \%)$ & $2(20 \%)$ & 0.203 & \\
\hline Combined* & $9(53 \%)$ & $3(30 \%)$ & 0.203 & \\
\hline Age (yr) & $28(24-31)$ & $31(23-39)$ & 0.458 & 1 \\
\hline Sex, males & $16(94 \%)$ & $7(70 \%)$ & 0.088 & \\
\hline RTS score & $0(0-0)$ & $3.92(3.39-6.38)$ & $<0.001$ & \\
\hline ISS & $34(27-51)$ & $25(16-26)$ & 0.003 & \\
\hline Ps & $0.01(0.00-0.03)$ & $0.74(0.31-0.94)$ & $<0.001$ & \\
\hline GCS score & $3(3-3)$ & $8(4-14)$ & $<0.001$ & \\
\hline $\begin{array}{l}\text { SOL at scene of } \\
\text { accident }\end{array}$ & $7(41 \%)$ & $10(100 \%)$ & 0.003 & 1 \\
\hline $\begin{array}{l}\text { Arrival ED after } \\
\text { injury (min) }\end{array}$ & $20(15-35)$ & $27(21-30)$ & 0.313 & 4 \\
\hline $\begin{array}{l}\mathrm{ET}<30 \text { min after } \\
\text { injury }\end{array}$ & 8 & 4 & 0.604 & 6 \\
\hline $\begin{array}{l}\mathrm{ET}>30 \text { min after } \\
\text { injury }\end{array}$ & 5 & 4 & & \\
\hline
\end{tabular}

More patients with penetrating injuries compared with those with blunt injuries survived $(37.0 \%$ vs. $12.2 \%, p=$ $0.004)$. Within the group of patients with penetrating injury (Table 4), survivors had a significantly lower ISS $(p=$ $0.003)$, higher RTS $(p<0.001)$, higher GCS score $(p<$ $0.001)$, and better PS $(p<0.001)$ compared with the non- 
TABLE 5. Predictors for Survival by Logistic Regression

\begin{tabular}{lcr}
\hline & \multicolumn{2}{c}{ Univariate Analysis } \\
\cline { 2 - 3 } Predictor & Odds Ratio (95\% CI) & $\boldsymbol{p}$ Value \\
\hline Penetrating & $4.3(1.5-11.8)$ & 0.006 \\
SOL at site & $5.67(0.7-45.0)$ & 0.101 \\
Sex, males & $0.69(0.23-2.08)$ & 0.510 \\
Age & $1.00(0.97-1.03)$ & 0.975 \\
GCS score $(>3)$ & $5.3(1.8-15.0)$ & $<0.001$ \\
ED arrival time & $1.00(0.99-1.00)$ & 0.400 \\
ET after injury $(<30 \mathrm{~min})$ & $1.0(0.3-3.2)$ & 0.994 \\
\hline
\end{tabular}

survivor group. No such difference was found between survivors/nonsurvivors in the blunt injury group.

Univariate logistic regression analysis (Table 5) showed that to be a victim of penetrating injury and to have a GCS score $>3$ were predictors of survival with odds ratios of 4.3 (95\% CI, 1.5-11.8) and 5.3 (95\% CI, 1.8-15), respectively. However, a forward stepwise multiple logistic regression model supplemented with interaction terms failed to reveal any predictors of survival.

\section{Long-term Outcome Data}

Of the surviving patients, 17 are neurologically intact $(85 \%)$. One patient died from septicaemia in another hospital after 51 days from septicaemia. The two remaining patients had blunt head injury. One of these developed a global cerebral edema and the other had a focal brain injury. The first was not extubated before the transfer to another country and the functional outcome is not known. The latter has over 2 years reported fatigue and concentration inhibition, despite intact cognitive abilities. In addition, two patients had severe orthopedic complications including amputation of one of the extremities. One patient with penetrating trauma, who underwent a median sternotomy, developed osteomyelitis of the sternum.

\section{DISCUSSION}

Our study shows that 20 of 109 patients subjected to ET in a Scandinavian hospital survived. A higher proportion of patients with penetrating (37.0\%) compared with blunt injury $(12.2 \%)$ survived.

The American College of Surgeons-Committee on Trauma reported an overall survival rate of $7.8 \%$ for ET patients in a meta-analysis from $2001 .{ }^{4}$ The study further showed that when outcome was stratified according to mechanism of injury, $11.2 \%$ and $1.6 \%$ of patients with penetrating and blunt injuries survived, respectively. ${ }^{4}$ The discrepancy between their and our results can be discussed in light of the following questions: (1) Does UUH have a too liberal protocol? i.e., is ET done on too healthy patients?; (2) Does UUH have a too selective ET protocol? i.e., is there avoidance of performing the procedure on the most injured; (3) Are survival rates in Europe and North America comparable?; and (4) Has a more formalized trauma system and improvement of trauma care led to better outcome for patients in the latter years?
The first question can be answered from a review of the patient data. PS, ISS, and RTS for the survivors at UUH were $0.54,26$, and 3.9 , respectively, which indicate severely traumatized patients. On the other hand, the relatively high median RTS may point toward a too liberal indication. A thorough investigation of all patients' records revealed, however, only one questionable ET. The patient, a 33-year-old man, was transferred from another hospital after he had bled $1.3 \mathrm{~L}$ from the chest tube. He had been stabbed and an explorative pericardiotomy was undertaken at UUS without findings of cardiac tamponade. Treatment with a chest tube would probably have been adequate.

In an attempt to answer the second question, all the patients who were declared dead within 1 hour after presentation at the ED and who did not undergo ET were investigated. There were 24 such patients. Thirteen were not candidates for ET (i.e., drowning, isolated head injury, burn injuries). Eight fulfilled the contraindications for ET as defined by the Trauma Manual of UUH, which leaves three patients. The first, an 88-year-old woman suffering from a blunt injury had on admission systolic blood pressure of $125 \mathrm{~mm} \mathrm{Hg}$ and a GCS score of 12 . However, she deteriorated rapidly and did not respond to fluid resuscitation. Because of the patient's old age, ET was considered futile. The second patient was a 24-year-old man with multiple self-inflicted stab wounds to the neck, elbow, and wrist. He had been without SOL at least 30 minutes before arrival to the ED and resuscitation was ended after 12 minutes. The argument for not initiating ET was the uncertainty of how long he had been without SOL. The final patient, a 31-year-old man with multiple gunshot wounds to the head, thorax, abdomen, and extremities arrived UUH 1 hour after injury. He had a GCS score of 3 and a systolic blood pressure of $60 \mathrm{~mm} \mathrm{Hg}$. Laparotomy and pericardiocentesis were performed. This patient could, in our opinion, have been a suitable candidate for ET before the extent of the head injury had been evaluated.

To answer the third question, further ET reports from European trauma centers must be provided. Based on these reports, a meta-analysis should be done. A comparison with literature from the United States should then be performed to rule out uncertainties. Agreement upon all data field definitions in participating trauma centers is a prerequisite to draw valid conclusions of such a study.

The final question will be answered by reports from comparable trauma centers. The meta-analysis that sets precedence today is based on reports from 1974 to $1998 .{ }^{4}$ Considering the improvement in pre, peri, and posthospital trauma care in the past decade, these numbers may not be accurate.

\section{Predictors of Outcome}

The contraindications for ET at UUH, as defined by the Trauma manual, were violated on 27 (24\%) occasions between 2001 and 2007. The patients were all suffering from blunt trauma and presented in the ED without SOL. Reasons for the attempt ranged from "not recognizing the severity of the injury" to "one last attempt." Interestingly, two of these patients $(7.4 \%)$ survived. Both had confirmed SOL at site but went into cardiac arrest $\sim 2$ and 5 minutes before arriving at the ED. The survival of these patients clarifies the need for a 
discussion on the validity of the contraindications at our hospital. Regardless of this, the cases illustrate the complexity of finding good predictors for outcome. In accordance with this, no predictors of outcome was found from the forward stepwise multiple regression analysis when analyzing the entire cohort and implementing interaction terms for suspected interactions between variables, especially between dominant mechanism of injury and other variables. In light of the univariate results, this suggest that perhaps two regression analysis should be conducted, one for blunt and one for penetrating trauma. This study has not enough patients for such analyses.

\section{Is ET Futile in a Scandinavian Trauma Center?}

Soreide et al. recently reported zero survivors out of 10 ET procedures during a 5-year period in a Norwegian Trauma Hospital The authors called for additional ET reports from Europe and Scandinavia and concluded that a reevaluation of the decision-making process was necessary. They further questioned if resuscitative emergency thoracotomies in a Scandinavian trauma hospital is justified. ${ }^{9}$ However, the results of Soreide et al. are not statistically inferior to our result as shown by Fischer's exact test (zero of 10 vs. 20 of 109 gives in a two-sided $p$ value of 0.209 ). The discrepancy between our two institutions results and conclusions clearly show, as Soreide et al. also states, that further ET studies are needed to rule out any statistical uncertainties. It also illustrates that if conclusions that could impact a protocol should be reached, they must be drawn under the assumption of comparability between the trauma hospitals. The study of Soreide et al. is listed as one of the five references in the 8th edition of the ATLS student manual revised ET segment. ${ }^{11}$ The difference between these references in regarding described methodology in general and training of trauma team, in particular, makes it difficult to extrapolate current conclusions beyond the respective institutions.

\section{Limitations}

The number of patients in this study is limited, and we do not have complete logistic data for all patients, which is a limitation of the study. Outcome is only based on survival status 30 days after injury.

\section{CONCLUSION}

It should, for a trained trauma team, be a liberal attitude toward performing an ET on the agonal patient. The ET procedure should be included as a necessary tool in the initial trauma treatment.

\section{ACKNOWLEDGMENTS}

We thank Morten Hestnes, RN, trauma registrar for his help in providing the data.

\section{REFERENCES}

1. Biffl WL, Moore EE, Johnson JL. Emergency department thoracotomy. In: Moore EE, Feliciano DV, Mattox KL, eds. Trauma. 5th ed. New York: McGraw-Hill 2004;239-254.

2. Rhee PM, Acosta J, Bridgeman A, Wang D, Jordan M, Rich N. Survival after emergency department thoracotomy: review of published data from the past 25 years. J Am Coll Surg. 2000;190:288-298.

3. Pillgram-Larsen J. Behandlinger. In: Høgevold $\mathrm{H}$, ed. Initialbehandling av den multitraumatiserte pasient ved Ullevål Universitetssykehus. Fresenius Kabi: Akuttstuegruppa, Ullevål Universitetssykehus; 2006:95-109.

4. Practice management guidelines for emergency department thoracotomy. Working Group, Ad Hoc Subcommittee on Outcomes, American College of Surgeons-Committee on Trauma. J Am Coll Surg. 2001;193: 303-309.

5. Miglietta MA, Robb TV, Eachempati SR, et al. Current opinion regarding indications for emergency department thoracotomy. J Trauma. 2001;51:670-676.

6. Rothenberg SS, Moore EE, Moore FA, Baxter BT, Moore JB, Cleveland HC. Emergency Department thoracotomy in children - a critical analysis. J Trauma. 1989;29:1322-1325.

7. Seamon MJ, Fisher CA, Gaughan JP, Kulp H, Dempsey DT, Goldberg AJ. Emergency department thoracotomy: survival of the least expected. World J Surg. 2008;32:604-612.

8. Seamon MJ, Pathak AS, Bradley KM, et al. Emergency department thoracotomy: still useful after abdominal exsanguination? J Trauma. 2008;64:1-7; discussion 7-8.

9. Soreide K, Soiland H, Lossius HM, Vetrhus M, Søreide JA, Søreide E. Resuscitative emergency thoracotomy in a Scandinavian trauma hospital - is it justified? Injury. 2007;38:34-42.

10. Baxter BT, Moore EE, Moore JB, Cleveland HC, McCroskey BL, Moore FA. Emergency department thoracotomy following injury: critical determinants for patient salvage. World J Surg. 1988;12:671675 .

11. Kortbeek JB, Al Turki SA, Ali J, et al. Advanced trauma life support, 8th edition, the evidence for change. J Trauma. 2008;64:1638-1650.

12. Fosse E, Pillgram-Larsen J, Rosen L. [Emergency thoracotomy. Aortic clamping in major bleeding]. Tidsskr Nor Laegeforen. 1991;111:26272629.

13. Osler T, Baker SP, Long W. A modification of the injury severity score that both improves accuracy and simplifies scoring. J Trauma. 1997;43: 922-925; discussion 925-926.

14. Stevenson M, Segui-Gomez M, Lescohier I, Di Scala C, McDonaldSmith G. An overview of the injury severity score and the new injury severity score. Inj Prev. 2001;7:10-13.

15. Champion HR, Sacco WJ, Copes WS, Gann DS, Gennarelli TA, Flanagan ME. A revision of the Trauma Score. J Trauma. 1989;29:623-629.

16. Association for the Advancement of Automotive Medicine. The Abbreviated Injury Scale. 1990 Revision. Des Plaines, IL: Association for the Advancement of Automotive Medicine; 1990.

17. Boyd CR, Tolson MA, Copes WS. Evaluating trauma care: the TRISS method. Trauma Score and the Injury Severity Score. J Trauma. 1987; 27:370-378.

18. Skaga NO, Eken T, Steen PA. Assessing quality of care in a trauma referral center: benchmarking performance by TRISS-based statistics or by analysis of stratified ISS data? J Trauma. 2006;60:538-547.

19. Champion HR, Sacco WJ, Copes WS. Injury severity scoring again. J Trauma. 1995;38:94-95.

20. Skaga NO, Eken T, Jones JM, Steen PA. Different definitions of patient outcome: consequences for performance analysis in trauma. Injury. 2008;39:612-622. 